This work needs to be replicated in other large longitudinal studies of both non-pregnant women and men.

J. Evans Division of Psychiatry, University of Bristol, Cotham House, Cotham Hill, Bristol BS6 6JL, UK.

E-mail: j.evans@bristol.ac.uk

J. Heron Unit of Paediatric and Perinatal

Epidemiology, Division of Child Health, University of Bristol

G. Lewis, R. Araya Division of Psychiatry, University of Bristol

D. Wolke Unit of Paediatric and Perinatal Epidemiology, Division of Child Health, University of Bristol

\section{Hypertension and depression in late life}

The 'vascular depression' hypothesis has attracted considerable interest, but its basis is by no means clear. Kaimal \& Nair (2005) in their recent correspondence mention vascular comorbidity in late-onset depression, citing a high prevalence rate of hypertension in particular. However, despite reasonably consistent findings of neuroradiological abnormalities associated with late-life depression, there is actually little evidence that hypertension or other 'traditional' vascular risk factors are responsible. What evidence there is for higher comorbidity comes from casecontrol studies comparing people with late-life depression who are known to clinical services with community controls; these studies carry a high risk of selection bias. Studies carried out in community populations have not generally found any associations between hypertension and depression (Kim et al, 2004), even in highrisk samples (Stewart et al, 2001).

The direction of causation between vascular disorders and depression is also unclear (Baldwin, 2005). Evidence for neuroradiological abnormalities associated with depression is derived almost entirely from cross-sectional studies, and there is currently much stronger prospective evidence that depression is a risk factor for vascular disorders than vice versa. The same appears to be the case for subsyndromal depressive symptoms that may not be recalled or reported in later life and might well have been present in people whose depression is classified as 'late-onset'. Neuropathological studies of late-life depression do not find the microvascular abnormalities that would be expected if hypertension were responsible, but instead find increased large vessel disease (Thomas et al, 2001), which could equally be a consequence as a cause of depression.

The uncertainty surrounding specific vascular processes in late-life depression is not just of academic interest, since there are important public health implications. Depression is strongly predicted by poor physical health and associated disability and there is little evidence at present to justify distinguishing specific disorders as responsible. The relationship between vascular processes and affective disorder is interesting, but there is a danger that more obvious risk factors for late-life depression (disability, poverty, loneliness) are ignored because they do not fall within the exciting world of organic psychiatry.

Baldwin, R. C. (2005) Is vascular depression a distinct sub-type of depressive disorder? A review of causal evidence. International Journal of Geriatric Psychiatry, 20 I-II.

Kaimal, A. B. \& Nair, U.V. (2005) Organic brain dysfunction in late-onset depression. British Journal of Psychiatry, I87, 288.

Kim, J.-M., Stewart, R., Shin, I. S., et al (2004) Vascular disease/risk and late-life depression in a Korean community population. British Journal of Psychiatry, 185, 102-107.

Stewart, R., Prince, M., Mann, A., et al (200I) Stroke, vascular risk factors and depression. Crosssectional study in a UK Caribbean-born population. British Journal of Psychiatry, 178, 23-28.

Thomas, A. J., Ferrier, I. N., Kalaria, R. N., et a (2001) A neuropathological study of vascular factors in late-life depression. Journal of Neurology, Neurosurgery and Psychiatry, 70, 83-87.

R. Stewart Section of Epidemiology, Institute of Psychiatry, King's College London, De Crespigny Park, London SE5 8AF, UK.

E-mail: r.stewart@iop.kcl.ac.uk

\section{Detection of depression in elderly care home residents}

Dr Eisses et al (2005) showed how the training of care home staff can help in detecting depression among elderly care home residents. In view of the high prevalence of depression in this group, these results are to be welcomed. However, I would like to raise a few points about the relevance of this report to care homes in England.

First, 8 out of 23 homes ( $35 \%$ ) declined to participate, citing lack of interest or feeling that it was too much work. This matches my own experience as a consultant old age psychiatrist dedicated to providing support to care homes with nursing. I would welcome any suggestions on how to engage homes in training to improve the detection of depression in this vulnerable population.

Second, I was impressed by the stability of the workforce, who had spent on average 9.5 years in the participating homes (the shortest period being 10 months). This is considerably longer than is found within some care homes in England, particularly in the large cities. It may be that training would be less effective when staff tend to have a higher rate of turnover.

Finally, I note that the study excluded residents with dementia who scored less than 15 out of 30 on the Mini-Mental State Examination. This decision is understandable as the scale used, the Geriatric Depression Scale (GDS), is difficult to administer to this group. However, I wonder about the effect on the staff. Training staff to exclude these residents from an assessment of depression could send a false signal that these individuals are somehow immune to depression - which most certainly is not the case. Also this approach probably could not apply in care homes with nursing in England. Whereas in the homes studied the prevalence of dementia was only some $9 \%$ of the population, dementia in care homes with nursing is high, with 'non-EMI' (elderly mental illness) care homes with nursing having a prevalence of dementia as high as $75 \%$ (Macdonald \& Carpenter, 2003). We use the Cornell Scale for Depression in Dementia (Alexopoulos et al, 1988) for those people with dementia who can not respond to the questions of the GDS.

Alexopoulos, G. S., Abrams, P. C., Young, R. C., et al (1988) Cornell Scale for Depression in Dementia. Biological Psychiatry, 23, 27I-284.

Eisses, A. M. H., Kluiter, H., Jongenelis, K., et al (2005) Care staff training in detection of depression in residential homes for the elderly. Randomised trial. British Journal of Psychiatry, 186, 404-409.

Macdonald, A. J. \& Carpenter, G. I. (2003) The recognition of dementia in 'non-EMl' nursing home residents in South East England. International Journal of Geriatric Psychiatry, 18, 105-108.

A. Thompsell Southwark Primary Care Trust, Care Homes Support Team, West Home, East Dulwich Grove, Dulwich Hospital, London SE22 8PT, UK.

E-mail: Amanda.thompsell@southwarkpct.nhs.uk 